\title{
BMJ Open Efficacy and safety of acupuncture as an adjuvant treatment for acute pancreatitis: a protocol of systematic review and meta-analysis
}

\author{
Kai Zhang, ${ }^{1}$ Chengyu Li, ${ }^{2}$ Chao Gao, ${ }^{3}$ Chen Zhao, ${ }^{4}$ Qilin Tang, ${ }^{5}$ Jingbo Zhai, ${ }^{6}$
} Yan $\mathrm{Li}^{1}$

To cite: Zhang K, Li C, Gao C, et al. Efficacy and safety of acupuncture as an adjuvant treatment for acute pancreatitis: a protocol of systematic review and meta-analysis. BMJ Open 2019;9:e029327. doi:10.1136/ bmjopen-2019-029327

- Prepublication history and additional material for this paper are available online. To view please visit the journal (http:// dx.doi.org/10.1136/bmjopen2019-029327).

$\mathrm{KZ}, \mathrm{CL}, \mathrm{CG}, \mathrm{CZ}$ and $\mathrm{QT}$ contributed equally.

Received 22 January 2019 Revised 9 April 2019 Accepted 12 June 2019
Check for updates

(C) Author(s) (or their employer(s)) 2019. Re-use permitted under CC BY-NC. No commercial re-use. See rights and permissions. Published by BMJ.

For numbered affiliations see end of article.

\section{Correspondence to}

Dr. Jingbo Zhai;

zhaijingbo@foxmail.com and

Dr Yan Li;

liyan66950@sina.com

\section{ABSTRACT}

Introduction Acupuncture may be effective for acute pancreatitis (AP). This systematic review aims to assess the efficacy and safety of acupuncture as an adjuvant treatment for AP.

Methods and analysis We will search PubMed, the Cochrane Central Register of Controlled Trials, EMBASE, Web of Science, China National Knowledge Infrastructure, Wan Fang Data and Chinese Science Journal Database from inception to 30 June 2019 to identify any eligible study. Only randomised controlled trials will be included. The selection of studies, data extraction and management will be completed by two reviewers independently. The primary outcomes include the overall response rate, mortality during the treatment, the proportion of patients with severe acute pancreatitis transferred to the intensive care unit or scheduled for surgery, gastrointestinal function and the acute physiology and chronic health evaluation II scores. The secondary outcomes include visual analogue scale, the use of analgesics, the recovery time of blood amylase becoming normal, tumour necrosis factor $\alpha$ counts, IL-6 counts, IL-10 counts, length of hospital stay and adverse events related to acupuncture (such as fainting, nausea, haematoma and local infection). Review Manager V.5.3 software will be used for statistical analyses. The risk of bias of included studies will be assessed by the Cochrane 'risk of bias' tool.

Ethics and dissemination This study will not involve personal information. The ethical approval will not be required. The results will be published in a peer-reviewed journal.

Trial registration number CRD42018115099

\section{INTRODUCTION}

Acute pancreatitis (AP) is an inflammatory injury of the pancreas caused by oedema, haemorrhage and necrosis associated with the digestion of pancreatic tissue. ${ }^{12} \mathrm{AP}$ is one of the most common acute abdominal diseases in clinical practice. ${ }^{34}$ Despite the rapid medical advancement in the last decade, the overall mortality of AP and severe acute pancreatitis (SAP) remains high. ${ }^{5-7}$ The gastrointestinal disorder is a common
Strengths and limitations of this study

- We will only include randomised controlled trials.

- Language and publication date will not be restricted.

- We will attempt to contact the original researchers by email to obtain any missing or inadequate data.

- We will conduct the sensitivity analysis to test whether the conclusions are robust.

- Different types of acupuncture therapy may lead to a large degree of heterogeneity.

clinical phenomenon in $\mathrm{AP}^{8}$ In the early stage of SAP, the bowel dysfunction is one of common symptoms, which may lead to the translocation of inflammatory mediators and toxic products. This eventually causes systemic inflammatory response syndrome (SIRS) and multiple organ dysfunction syndrome (MODS).$^{9-11}$ Therefore, improving the recovery of gastrointestinal function is very important for patients with AP. Some studies showed the efficacy of acupuncture for gastrointestinal disorders. ${ }^{12} 13$

Opioids are safe and effective in treating AP patients. Compared with other analgesic drugs, opioids can reduce the need for supplemental analgesia. ${ }^{14}$ However, frequent use of opioid analgesics may lead to opioid dependence. ${ }^{15}$ One of the most important applications of acupuncture is for pain relief. ${ }^{16}$ The analgesic effect of acupuncture is mainly achieved by the regulation of the central nervous system. Substances such as enkephalin, dynorphin, 5-hydroxytryptophan, epinephrine and somatostatin produced in the spinal cord may contribute to analgesic effects of acupuncture. ${ }^{17} 18$ Acupuncture may be a suitable alternative to opioid analgesics because it is considered an effective analgesic therapy without dependence.

The morbidity and mortality of AP depend on the severity and balance of 
the inflammatory response. ${ }^{19}$ In the early stage of SAP, pancreatic enzymes are activated and released into the blood, which activate monocytes/macrophages and release a large number of proinflammatory cytokines, such as tumour necrosis factor $\alpha$ (TNF- $\alpha)$, IL- 6 and so on. They can stimulate various inflammatory cells and cause an overactive proinflammatory response. This leads to an uncontrolled systemic response that ultimately results in SIRS and MODS. ${ }^{19}{ }^{20}$ IL-6 can not only directly damage vascular endothelial cells and activate inflammatory cells but also aggravate inflammatory reactions. However, the self-protection mechanism increases the anti-inflammatory factor IL-10 to inhibit the synthesis of the proinflammatory factors mentioned above, and finally reduces the systemic inflammatory response. Studies have shown that inhibiting excessive inflammatory responses is the key to the treatment of AP. Changes in serum IL- 6 and IL-10 can be used as indicators to judge the severity of AP and evaluate the prognosis. ${ }^{21}$

Acupuncture can reduce proinflammatory mediators in the process of inflammatory response. ${ }^{22}$ An experiment found that electroacupuncture could reduce TNF- $\alpha$ and IL-6 in rats. ${ }^{23}$ Another study showed that acupuncture might have therapeutic effects on AP by inhibiting nuclear factor $\mathrm{KB}$ expression and reducting the release of proinflammatory cytokines. ${ }^{24}$ Some studies found that electroacupuncture could decrease TNF- $\alpha$, IL- 6 and increase IL-10 to reestablish the balance between proinflammatory and anti-inflammatory cytokines, and reduce the inflammatory response in AP. ${ }^{25-27}$

To our knowledge, there is a lack of high-quality evidence on acupuncture in the treatment of AP. Thus, this systematic review aims to assess the efficacy and safety of acupuncture as an adjuvant treatment for AP.

\section{METHODS}

\section{Criteria for including studies in the review}

Types of studies

We will only include randomised controlled trials (RCTs) of acupuncture therapy for AP regardless of any language or publication status. We will also remove studies without comparable baselines and duplicate publications.

\section{Types of participants}

Participants diagnosed with AP according to the internationally acknowledged diagnostic criteria for AP will be included. These criteria include the revised Atlanta classification of AP, American clinical guideline for AP, the clinical diagnostic criteria for AP developed by the pancreatic division of the surgery division of the Chinese medical association in 1996 or 2014, the diagnostic and therapeutic guideline for SAP in 2001, and the diagnostic and therapeutic guideline for AP in China in 2004. ${ }^{28-31}$ All of the participants should receive acupuncture treatment and be hospitalised within 48 hours of symptom onset. There is no restriction on age, gender or ethnicity of the enrolled subjects.

\section{Types of outcomes}

\section{The primary outcomes}

The primary outcomes include the overall response rate, mortality during treatment, the proportion of SAP patients transferred to the intensive care unit (ICU) or scheduled for surgery, gastrointestinal function and the acute physiology and chronic health evaluation (APACHE) II scores.

The overall response rate is computed based on the diagnostic standard in the draft of diagnosis and treatment of AP proposed by the pancreas group of the surgical society of China medical association in $2000 .^{32}$ It is divided into four grades: cure, obvious effect, effective and ineffective. It can also be computed based on the 2002 edition of the guideline for clinical researches of traditional Chinese medicine new drugs. ${ }^{27}$ It is divided into three levels: obvious effect, effective and ineffective.

The proportion of SAP patients transferred to the ICU or scheduled for surgery: it is difficult to treat SAP patients with conservative therapies in the general ward. SIRS and MODS could exacerbate SAP symptoms. Therefore, some SAP patients could be transferred to ICU or scheduled for surgery.

Gastrointestinal function includes abdominal pain and distension relief, recovery time of bowel sound, recovery time of defecation and time of resuming to diets (days).

APACHE II is a useful prognostic scoring system for predicting the severity of AP and can be of vital importance in determining the group of patients who have more chance of requiring tertiary care in the course of treatment. $^{33} 34$

\section{The secondary outcomes}

The secondary outcomes include visual analogue scale, the use of analgesics, the recovery time of blood amylase becoming normal, TNF- $\alpha$ counts, IL- 6 counts, IL-10 counts, length of hospital stay and adverse events related to acupuncture (such as fainting, nausea, haematoma and local infection).

\section{Types of interventions}

We will perform a systematic review and meta-analysis that includes all RCTs using acupuncture plus routine treatment (RT) in the treatment group and RT alone in the control group. RT includes fluid resuscitation, use of antibiotics, nutritional support and mechanical ventilation and so on.

\section{Search strategy}

\section{Electronic searches}

We will search the following electronic databases from inception to 30 June 2019: PubMed, the Cochrane Central Register of Controlled Trials, EMBASE, Web of Science, China National Knowledge Infrastructure, Wan Fang Data and Chinese Science Journal Database. The searching strategy is available at appendix 1 . No language or publication date will be restricted. 


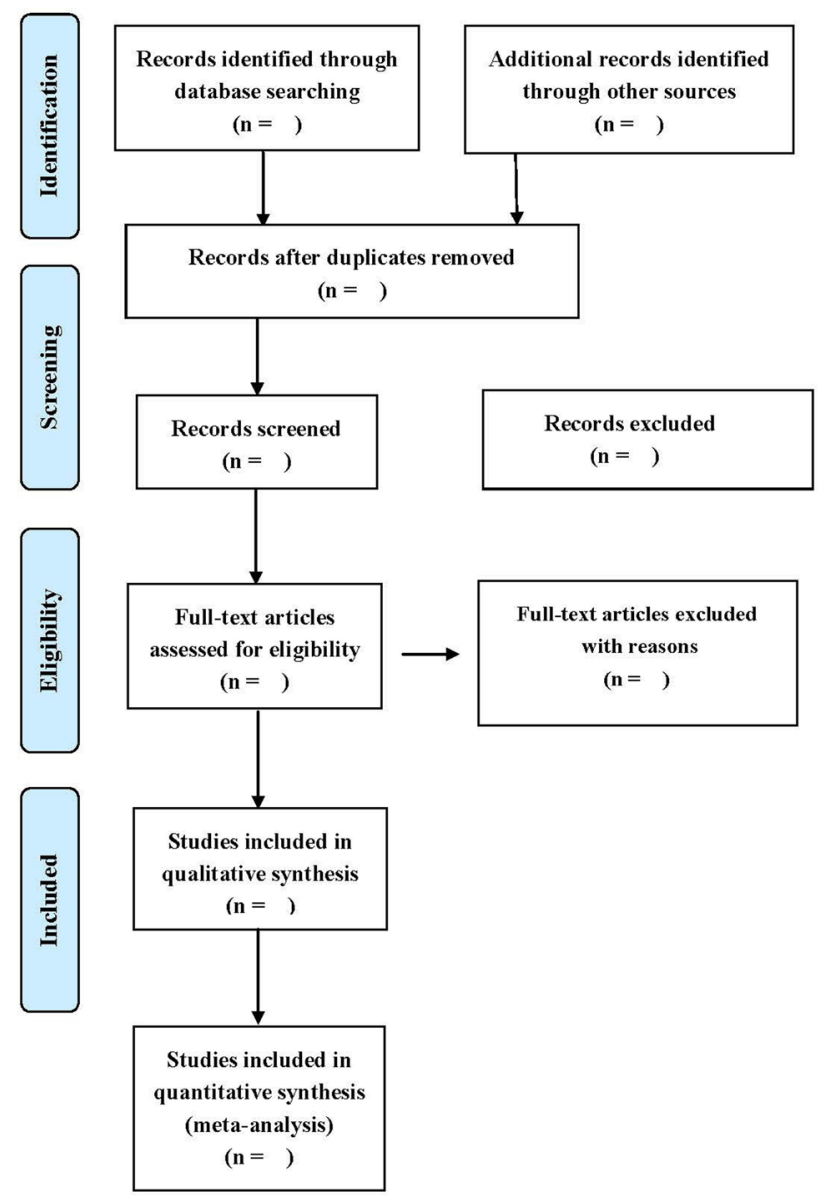

Figure 1 Study selection flow diagram.

\section{Searching other resources}

The reference lists of identified relevant RCTs, comments, reviews and overviews will be screened for additional relevant articles. The WHO International Clinical Trials Registry Platform, Chinese Clinical Trial Registry, Google Scholar and ClinicalTrials.gov will also be searched to identify any planned, ongoing or unpublished literature.

\section{Data extraction and management \\ Selection of studies}

The selection of studies will be completed independently by two reviewers (CG and KZ). They will check results with each other. When disagreements occur, a third reviewer (QT) will make the final decision. Full texts of all included studies will be read if necessary. Details of the selection process will be shown in the Preferred Reporting Items for Systematic Reviews and Meta-Analyses flowchart (figure 1).

\section{Data extraction and management}

A piloted data extraction form has been developed by reviewers. The following information will be extracted from each included study independently by two authors (QT and KZ): general information, study population, intervention characteristics and outcomes. Any disagreement will be resolved by discussion. If the information is unclear, missing or presented in a form that cannot be extracted, we will contact the corresponding author for additional information.

\section{Risk of bias assessment}

Two reviewers ( $\mathrm{KZ}$ and $\mathrm{CL}$ ) will independently assess the risk of bias using the Cochrane 'risk of bias' tool. ${ }^{35} 36$ This tool includes the following seven domains: (1) random sequence generation (selection bias); (2) allocation concealment (selection bias); (3) blinding of participants and personnel (performance bias); (4) blinding of outcome assessment (detection bias); (5) incomplete outcome data (attrition bias); (6) selective reporting (reporting bias); (7) other bias. The risk of bias for each domain will be graded high, low or unclear based on the relevant information extracted from each eligible study. Any disagreement will be resolved by consensus.

\section{Measures of treatment effect}

For continuous data, mean difference (MD) or standard MD (SMD) will be used to measure the therapeutic effect with $95 \%$ CIs. Risk ratios (RR) with $95 \%$ CIs will be calculated for dichotomous data.

\section{Dealing with missing data}

We will attempt to contact the original researchers by email to obtain any missing or inadequate data if possible. We will also obtain data by phone. If we cannot obtain accurate data through the above methods, we will exclude these studies.

\section{Assessment of heterogeneity}

The heterogeneity will be tested by the $\chi^{2}$ test or $\mathrm{I}^{2}$ value. If the $\mathrm{p}$ value in $\chi^{2}$ test is $<0.10$ or $\mathrm{I}^{2}$ is $>50 \%$, the heterogeneity across studies will be statistically significant. When the heterogeneity is identified, a meta-analysis with the random-effect model will be used to estimate overall treatment effect. Moreover, a subgroup analysis or meta-regression will be conducted to explore the causes of heterogeneity among results of studies.

\section{Assessment of reporting biases}

We will assess publication bias using the funnel plot and Egger's test if $>10$ trials are included.

\section{Data synthesis}

The meta-analysis will be conducted using the Review Manager V.5.3 software. We will describe the effect size with RR for dichotomous data, and MD or SMD for continuous data. If $\mathrm{I}^{2}$ is $<50 \%$, the fixed-effects model will be used for data synthesis. ${ }^{37} 38$ If $^{2}$ is $>50 \%$, we will combine the data using random-effects model. ${ }^{37}{ }^{38} \mathrm{~A}$ narrative description of the results will be conducted when the meta-analysis is not feasible.

\section{Subgroup analysis}

We will perform subgroup analysis to explore possible causes of heterogeneity if necessary. Subgroup analysis will be conducted based on the type of acupuncture 
intervention (body acupuncture, electroacupuncture, fire needling, elongated needle, warming needling), acupuncture point (single or multiple points), the duration of treatment, age and gender if possible.

\section{Sensitivity analysis}

We will conduct sensitivity analyses to test the robustness of pooled treatment effects. The following factors will be taken into consideration:

1. Impact of high or unclear risk of bias: excluding studies with high or unclear risk of bias for certain domain, such as random sequence generation.

2. Impact of selected models: fixed-effects model versus random-effects model.

If inconsistent results are identified, caution will be taken in interpreting results and drawing conclusions.

\section{Assessment method of evidence quality}

The quality of the evidence will be assessed using the Grading of Recommendations Assessment, Development and Evaluation. ${ }^{35}$ The evaluation items include risk of bias, inconsistency, indirectness, imprecision and publication bias. Two authors (KZ and CG) will independently assess the quality of the evidence based on five levels of criteria. It will fall into one of four possible ratings: high, moderate, low and very low.

\section{Ethics and dissemination}

This study will not involve personal information from individuals or violate their personal rights. Therefore, ethical approval will not be required. The results will be published in a peer-reviewed journal. Due to the lack of relevant systematic reviews in this field, this study will combine relevant RCTs to better explore evidence on acupuncture treatment for AP and guide clinical practice and acupuncture researches.

\section{Patient and public involvement}

Patients' priorities, experience and preferences were not involved in development of the research question, and outcome measures, the design of this study, or the recruitment to and conduct of the study. The results will be not disseminated to study participants.

\section{Amendments}

If the protocol is modified, the information will be described in the final report.

\section{DISCUSSION}

Acupuncture may be effective for AP. However, no systematic reviews on this topic have been published. This study will provide more convincing information on acupuncture for AP. This study has some potential limitations. Different types of acupuncture therapy may lead to a large degree of heterogeneity.

\section{Author affiliations}

${ }^{1}$ Department of Acupuncture and Moxibustion, Tianjin Gong An Hospital, Tianjin, China
${ }^{2}$ Key Laboratory of Chinese Internal Medicine of Ministry of Education and Beijing, Beijing University of Chinese Medicine Affiliated Dongzhimen Hospital, Beijing, China

${ }^{3}$ Tianjin Institute of General Surgery, Tianjin Medical University General Hospital, Tianjin, China

${ }^{4}$ Institute of Basic Research in Clinical Medicine, China Academy of Chinese Medical Sciences, Beijing, China

${ }^{5}$ Graduate College, Tianjin University of Traditional Chinese Medicine, Tianjin, China ${ }^{6}$ Institute of Traditional Chinese Medicine, Tianjin University of Traditional Chinese Medicine, Tianjin, China

Contributors $\mathrm{YL}, \mathrm{JZ}$ and $\mathrm{CZ}$ conceived the study and provided general guidance to the drafting of the protocol. CG and KZ drafted the protocol. $C L$ designed the search strategy. CG, KZ, CL, QT and CZ drafted the manuscript. CG, KZ, CL, CZ, JZ, YL and QT reviewed and revised the manuscript. All authors have read and approved the final version of the manuscript.

Funding The authors have not declared a specific grant for this research from any funding agency in the public, commercial or not-for-profit sectors.

Competing interests None declared.

Patient consent for publication Not required.

Provenance and peer review Not commissioned; externally peer reviewed.

Open access This is an open access article distributed in accordance with the Creative Commons Attribution Non Commercial (CC BY-NC 4.0) license, which permits others to distribute, remix, adapt, build upon this work non-commercially, and license their derivative works on different terms, provided the original work is properly cited, appropriate credit is given, any changes made indicated, and the use is non-commercial. See: http://creativecommons.org/licenses/by-nc/4.0/.

\section{REFERENCES}

1. $\mathrm{Xu}$ WB, $\mathrm{Hu} \mathrm{QH}, \mathrm{Wu} \mathrm{CN}$, et al. Serum soluble fibrinogen-like protein 2 concentration predicts delirium after acute pancreatitis. Brain Behav 2019;9:e01261.

2. Sarri G, Guo Y, Iheanacho I, et al. Moderately severe and severe acute pancreatitis : a systematic review of the outcomes in the USA and European Union-5. BMJ Open Gastroenterol 2019;6:e000248.

3. Forsmark CE, Swaroop Vege S, Wilcox CM. Acute Pancreatitis. N Engl J Med Overseas Ed 2016;375:1972-81.

4. Lankisch PG, Apte M, Banks PA. Acute pancreatitis. Lancet 2015;386:85-96.

5. Li Y, Zhao Y, Feng L, et al. Comparison of the prognostic values of inflammation markers in patients with acute pancreatitis: a retrospective cohort study. BMJ Open 2017;7:e013206.

6. van Dijk SM, Hallensleben NDL, van Santvoort HC, et al. Acute pancreatitis: recent advances through randomised trials. Gut 2017;66:2024-32.

7. Waller A, Long B, Koyfman A, et al. Acute Pancreatitis: updates for Emergency Clinicians. J Emerg Med 2018;55:769-79.

8. Wang X, Gong Z, Wu K, et al. Gastrointestinal dysmotility in patients with acute pancreatitis. J Gastroenterol Hepatol 2003;18:57-62.

9. Singh VK, Wu BU, Bollen TL, et al. Early systemic inflammatory response syndrome is associated with severe acute pancreatitis. Clin Gastroenterol Hepatol 2009;7:1247-51.

10. Halonen KI, Pettilä V, Leppäniemi AK, et al. Multiple organ dysfunction associated with severe acute pancreatitis. Crit Care Med 2002;30:1274-9.

11. McKay CJ, Buter A. Natural history of organ failure in acute pancreatitis. Pancreatology 2003;3:111-4.

12. Diehl DL. Acupuncture for gastrointestinal and hepatobiliary disorders. J Altern Complement Med 1999;5:27-45.

13. Wang CW, Li N, He HB, et al. [Effect of electroacupuncture of Tianshu (ST 25) on the rational symptoms of functional constipation patients and evaluation on its efficacy satisfaction: a single-center, prospective, practical and randomized control trial]. Zhen Ci Yan Jiu 2010;35:375-9.

14. Basurto Ona X, Rigau Comas D, Urrútia G. Opioids for acute pancreatitis pain. Cochrane Database Syst Rev 2013:CD009179.

15. Minozzi S, Amato L, Davoli M. Development of dependence following treatment with opioid analgesics for pain relief: a systematic review. Addiction 2013;108:688-98.

16. Vanderploeg K, Yi X. Acupuncture in modern society. $J$ Acupunct Meridian Stud 2009;2:26-33. 
17. Felson DT, Lawrence RC, Hochberg MC, et al. Osteoarthritis: new insights. Part 2: treatment approaches. Ann Intern Med 2000;133:726-37.

18. Andersson S, Lundeberg T. Acupuncture--from empiricism to science: functional background to acupuncture effects in pain and disease. Med Hypotheses 1995;45:271-81.

19. Cosen-Binker LI, Binker MG, Negri G, et al. Acute pancreatitis possible initial triggering mechanism and prophylaxis. Pancreatology 2003;3:445-56.

20. Winsö O, Kral J, Wang W, et al. Thoracic epidural anaesthesia reduces insulin resistance and inflammatory response in experimental acute pancreatitis. Ups J Med Sci 2018;123:207-15.

21. Pooran N, Indaram A, Singh P, et al. Cytokines (IL-6, IL-8, TNF) early and reliable predictors of severe acute pancreatitis. J Clin Gastroenterol 2003;37:263-6.

22. Xue QM, Huang L, Li N. [Effects of electroacupuncture at Tianshu (ST25) on pro- and anti-inflammatory cytokines in rats with severe acute pancreatitis]. Zhong Xi Yi Jie He Xue Bao 2011;9:658-64.

23. Torres-Rosas R, Yehia G, Peña G, et al. Dopamine mediates vagal modulation of the immune system by electroacupuncture. Nat Med 2014:20:291-5

24. Xue QM, Pan H, Huang L, et al. Effects of acupuncture at ST25 on inflammatory mediators and nuclear factor $\mathrm{\kappa B}$ activation in a rat model of severe acute pancreatitis. Acupunct Med 2015;33:299-304.

25. Zhu SF, Guo H, Zhang RR, et al. Effect of electroacupuncture on the inflammatory response in patients with acute pancreatitis: an exploratory study. Acupunct Med 2015;33:115-20.

26. Li L, Yu J, Mu R, et al. Clinical effect of electroacupuncture on lung injury patients caused by severe acute pancreatitis. Evid Based Complement Alternat Med 2017;2017:1-6.

27. Luo YH, Zhong GW, Zhao SP, et al. [Efficacy observation of electroacupuncture intervention on severe acute pancreatitis at early stage complicated with intestinal paralysis]. Zhongguo Zhen Jiu 2011;31:105-9.
28. Banks PA, Bollen TL, Dervenis C, et al. Classification of acute pancreatitis--2012: revision of the Atlanta classification and definitions by international consensus. Gut 2013;62:102-11.

29. Munigala S, Subramaniam D, Subramaniam DP, et al. Predictors for early readmission in acute pancreatitis (AP) in the United States (US) - A nationwide population based study. Pancreatology 2017:17:534-42.

30. Zhao YP. [Clinical importance of guideline for severe acute pancreatitis of China]. Zhonghua Wai Ke Za Zhi 2007;45:721.

31. Sarr MG. 2012 revision of the Atlanta classification of acute pancreatitis. Pol Arch Med Wewn 2013;123:118-24.

32. Wang $X Y$. [Electroacupuncture for treatment of acute pancreatitis and its effect on the intestinal permeability of the patient]. Zhongguo Zhen Jiu 2007;27:421-3.

33. Harshit Kumar A, Singh Griwan M. A comparison of APACHE II, BISAP, Ranson's score and modified CTSI in predicting the severity of acute pancreatitis based on the 2012 revised Atlanta Classification. Gastroenterol Rep 2018;6:127-31.

34. Rathnakar SK, Vishnu VH, Muniyappa S, et al. Accuracy and Predictability of PANC-3 Scoring System over APACHE II in acute pancreatitis: a prospective study. J Clin Diagn Res 2017;11:PC10-PC13.

35. Chandler J, Higgins JPT, Deeks JJ, et al. Cochrane Handbook for Systematic Reviews of Interventions Version 5.2.0, 2017.

36. Zeng X, Zhang Y, Kwong JS, et al. The methodological quality assessment tools for preclinical and clinical studies, systematic review and meta-analysis, and clinical practice guideline: a systematic review. J Evid Based Med 2015;8:2-10

37. Huang W, Yang Z, Tang D, et al. Pharmacological intervention of traditional Chinese medicine for the quality of life in patients with colorectal cancer: a systematic review and meta-analysis. Traditional Medicine Research 2018;3:95-105.

38. Chen $\mathrm{Y}$, Jin $\mathrm{Y}$, Fan $\mathrm{Y}$, et al. Meta-analysis of neostigmine injections given at the Zusanli (ST 36) acupoint in the treatment of postpartum urinary retention. Traditional Medicine Research 2018;3:106-14. 